\title{
The Pan London Emergency Cardiac Surgery Service Blueprint
}

\author{
Daniel Fudulu ${ }^{1}$, Gianni Angelini² ${ }^{2}$ and Hunaid Vohra ${ }^{1}$ \\ ${ }^{1}$ Bristol Heart Institute \\ ${ }^{2}$ Bristol University
}

May 29, 2020

\begin{abstract}
The authors share their experience of managing the cardiac surgery services across London during the challenging Covid-19 pandemic. The Pan London Emergency Cardiac Surgery Service model could serve as a blueprint to design policies applicable to other surgical specialities and parts of the UK and worldwide.
\end{abstract}

\begin{abstract}
The authors share their experience of managing the cardiac surgery services across London during the challenging Covid-19 pandemic. The Pan London Emergency Cardiac Surgery Service model could serve as a blueprint to design policies applicable to other surgical specialities and parts of the UK and worldwide.

Hussain et al. ${ }^{1}$ must be congratulated on setting up of the Pan London Emergency Cardiac Surgery Service PLECS in managing cardiac surgical patients during the unprecedented Covid-19 crisis. Undoubtedly, the speciality of cardiac surgery in London is in a unique position to develop such a protocol since London is one of the most affected and challenged cities in the world from the outset, contributing predominantly to Covid-19-related UK mortality which is the highest in Europe ${ }^{2}$. The graph for the number of people infected with Covid-19 admitted to a UK hospital reached its peak in the early April ${ }^{3}$. As of $18^{\text {th }}$ of May, London still leads with a total of 26440 confirmed Covid-19 cases $^{4}$. One of the critical strategies for managing such a public health emergency is careful containment. Therefore, the designation of Covid-free hospitals ("delivery units") that could perform cardiac surgery in a safe environment is paramount. Hence, centralisation of the cardiac surgery services from seven to two units with minimal Covid-19 contamination that could deal with London cardiac surgery pool of patients was one of the primary measures reported by the authors. Indeed, London is the only city in the UK which has the privilege to take such a step due to the number of cardiac units, manpower provision, resource allocation and transport infrastructure. However, the disruption of the ample provision of the above resources available normally, could, on the contrary, lead to worse chaos in a pandemic crisis in a city like London.

Maintenance of a Covid-19 free environment is equally important. This has now become an integral part of all decision tree algorithms throughout the world ${ }^{5}$. Broadly, this requires clinical screening and a Covid-19 nasopharyngeal swab test before and after the transfer of the patient for urgent and emergency surgery. Recently published national guidance ${ }^{6}$ suggests that computer tomography for screening for Covid-19 in elective patients (this would include urgent patients admitted from home with critical/life-threatening anatomy with worsening symptoms or the need for prognostic intervention) is not routinely recommended and should only be reserved for urgent or emergency cases. One can imagine that the breakout of Covid-19 in these two cardiac surgical units would lead to disastrous consequences. It is widely believed that patients with Covid-19 who undergo cardiac surgery have significantly higher morbidity and mortality. A retrospective cohort study of 34 elective general surgical operations on patients with confirmed Covid-19, $44.1 \%$ of patients, needed ICU care, and the mortality rate was $20.5 \%^{7}$.
\end{abstract}


It was with great regret that we recently learned about the demise of two UK cardiac surgeons with Covid-19. Incentives like the PLECS referral pathway have led to a heightened awareness of Covid-19 risk of infection in cardiac surgical practice in a relatively short time period. Such algorithms ensure health care worker safety but also can inform decision making when taking a patient for surgery.

The current article also highlights the need for hospitals to adopt theatre specific pathways with the assumption that all patients are Covid-19 positive despite a negative swab (false negative) ${ }^{5,8}$. While appropriate theatre personal protective equipment (PPE) is vital to ensure staff and patients safety, performing heart operations with full PPE (including respirator hoods) can be difficult and is associated with difficult vision, headaches, inadequate hearing/communication, facial pain and dry throat. This, along with the sheer nature of these advanced cardiac patients (older patients, critical disease, ongoing symptoms, hypertrophic and impaired left ventricle, emergency), makes cardiac surgery a challenging venture. Leadership based on hierarchical command is essential during such a crisis, akin to a war situation. The establishment of a command centre that uniquely coordinates cardiac surgical activity in the whole of London accordingly between the delivery unit and "referring" unit is of paramount significance for the smooth running of a crucial service in the capital. Many units throughout the UK have now adopted a Covid-19 central command hub similar to the one presented by the authors, mainly for Covid-19 in general. To assist in the allocation and timing of appropriate procedures, all cardiac surgical departments in the UK have embraced the concept of regular as well as adhoc multidisciplinary team meetings with the help of online conference tools. To avoid cardiac surgery-associated Covid-19 morbidity and mortality, TAVI or PCI is the recommended option if it is considered a reasonable treatment but not the usual recommended treatment as dictated by the best evidence. This would also avoid death on the surgical waiting list and minimise the burden on critical care.

Coming out of this pandemic, the focus will be on the need for risk stratifying patients referred for surgery and those on the already prolonged waiting list. Step-wise guidance for return of services dealing with cardiovascular diseases in North America has already been published ${ }^{9}$. It is estimated that 28,404,603 elective operations would have been cancelled worldwide during a 12-week peak disruption during the pandemic. Intelligent and sensible triaging of patients waiting to undergo cardiac surgery will test our leadership during a period of already pressurised health service. The usefulness PLECS Service model will be instrumental in serving as a blueprint to design policies applicable to other surgical specialities and parts of the UK and worldwide. However, due to the differing landscape of other UK cities, the PLECS model may not be entirely reproducible as in London and will have to be adapted to the regional characteristics. It would be of great interest to determine the characteristics, volume and outcomes of patients who have undergone urgent and emergency cardiac surgery under the PLEC service and a publication in this regard would be eagerly awaited.

\section{References:}

1. Hussain A, Damian B, Martin Y, et al. The Pan London Emergency Cardiac Surgery Service: Coordinating 4 a response to the COVID-19 pandemic. Journal Card Surg (in press) . 2020.

2. WHO Coronavirus Disease (COVID-19) Dashboard. https://covid19.who.int/. Accessed May 20, 2020.

3. Prime Minister's Office 10 Downing Street. Slides to Accompany Coronavirus Press Conference: 30 April 2020 .; 2020. https://www.gov.uk/government/publications/slides-and-datasets-to-accompany-coronaviruspress-conference-30-april-2020.

4. Coronavirus (COVID-19) in the UK. https://coronavirus.data.gov.uk/\#category=regions\&map=rate. Accessed May 18, 2020.

5. Forrester JD, Nassar AK, Maggio PM, Hawn MT. Precautions for Operating Room Team Members During the COVID-19 Pandemic. J Am Coll Surg . 2020. doi:10.1016/j.jamcollsurg.2020.03.030

6. Current Recommendations Regarding Pre-Operative COVID-19 Screening CT Scan in Patients Undergoing Cardiothoracic Surgery .; 2020. https://scts.org/covid-19/. 
7. Nepogodiev D, Bhangu A. Elective surgery cancellations due to the COVID-19 pandemic: global predictive modelling to inform surgical recovery plans. Br J Surg. May 2020. doi:10.1002/bjs.11746

8. SCTS/ACTACC/SCPS Theatre COVID Pathway ; $2020 . \quad$ https://scts.org/wpcontent/uploads/2020/03/SCTS-ACTACC-SCPS-Theatre-COVID-pathway-Final.pdf.

9. Virani A, Poppas A, Russo M, et al. Safe Reintroduction of Cardiovascular Services during the COVID-19 Pandemic: Guidance from North American Societies. Can J Cardiol . 2020. doi:10.1016/j.cjca.2020.04.031 